\title{
Local Scale Controlled Anisotropic Diffusion with Local Noise Estimate for Image Smoothing and Edge Detection
}

\author{
Ping Liang \\ College of Engineering \\ University of California \\ Riverside, CA 92521-0425 \\ liang@engr.ucr.edu
}

\author{
Y. F. Wang \\ Department of Computer Science \\ University of California \\ Santa Barbara, CA 93106 \\ yf wang@cs. ucsb.edu
}

\begin{abstract}
A novel local scale controlled piecewise linear diffusion for selective smoothing and edge detection is presented. The diffusion stops at the place and time determined by the minimum reliable local scale and a spatial variant, anisotropic local noise estimate. It shows nisotropic, nonlinear diffusion equation using diffusion coefficients/tensors that continuously depend on the gradient is not necessary to achieve sharp, undistorted, stable edge detection across many scales. The new diffusion is anisotropic and asymmetric only at places it needs to be, i.e., at significant edges. It not only does not diffuse across significant edges, but also enhances edges. It advances geometry-driven diffusion because it is a piecewise linear model rather than a full nonlinear model, thus it is simple to implement and analyze, and avoids the difficulties and problems associated with nonlinear diffusion. It advances local scale control by introducing spatial variant, anisotropic local noise estimation, and local stopping of diffusion. The original local scale control was based on the unrealistic assumption of uniformly distributed noise independent of the image signal. The local noise estimate signifcantly improves local scale control.
\end{abstract}

\section{Introduction}

The theory of linear scale-space $[6,12]$ enables the detection and localization of edges while eliminating noise by tracking features across multiple scales. Koenderink pointed out that linear-scale space is equivalent to linear diffusion [6]:

$$
\begin{array}{r}
\frac{\partial u(x, y, t)}{\partial t}=\Delta u(x, y, t),(x, y) \in \Omega, t>0 \\
u(x, y, 0)=u_{0}(x, y),(x, y) \in \Omega \\
\frac{\partial u}{\partial e_{n}}=0,(x, y) \in \partial \Omega, t>0
\end{array}
$$

where $u_{0}(x, y)$ is the original image, $\frac{\partial u}{\partial e_{n}}$ is the directional derivative of $u$ in the normal direction of the boundary of $\Omega$, and $\sqrt{t}$ is the scale factor. Linear diffusion smoothes out noise and edges equally. After a few iterations of linear diffusion, all edges are smeared and distorted. This distortion causes difficulty in tracking features across multiple scales.

Perona and Malik [7, 8] first introduced the idea of nonlinear diffusion where diffusion is preferred within a smooth region to diffusion near an edge. They replaced the homogeneous heat equation (2) by a nonlinear equation

$$
\frac{\partial u}{\partial t}=\operatorname{div}(g(|\nabla u|) \nabla u)
$$

where $g$ is a smooth nonincreasing function with $g(0)=1, g(s) \geq 0, s \geq 0$ and $g(s) \rightarrow 0$ when $s \rightarrow \infty$. This actually introduces part of edge detection into the diffusion process, and allows interaction between scales right from the beginning of the diffusion process. In the nonlinear diffusion process by Perona and Malik, edges remain sharp and undistorted across many scales. Thus it yields stable edges across many scales and makes tracking features between scales unnecessary. However, the nonlinear diffusion process introduced by Perona and Malik does not perform well with noisy images and very similar images could produce divergent solutions, as pointed out in $[3,1]$. The problem is mainly due to the dependence of the variable diffusion coefficient $g(|\nabla u|)$ on the magnitude of the gradient. Lions and Morel's group [3] proposed to solve the problem by replacing the gradient inside $g(|\nabla u|)$ with the estimate from a smoothed image, i.e., replacing $g(|\nabla u|)$ with $g\left(\left|\nabla G_{\sigma} * u\right|\right)$. Thus, an image is first smoothed by convolving with a Gaussian kernel of size $\sigma$. It can be shown that this simple modification solves the difficulties with the Perona and Malik formulation. A question that needs to be answered before applying the diffusion process is what size Gaussian kernel should be used to smooth the image. Note 
that the whole image will be smoothed by a Gaussian kernel of the same size.

Both diffusion processes in Eq. (2) and in [3] are inhomogeneous, but still isotropic. This is because the diffusion coefficients are still a scalar. Note that although their formulation was isotropic, Perona and Malik actually implemented an anisotropic diffusion process by making the diffusion coefficient in each of the four diffusing directions depend on the directional gradient in that direction [7, 8]. Lions and Morel's group further extended their work by modifying the diffusion operator [1]:

$$
\frac{\partial u}{\partial t}=g\left(\left|\nabla G_{\sigma} * u\right|\right)|\nabla u| \operatorname{div} \frac{\nabla u}{|\nabla u|},
$$

This process is anisotropic and has a geometric interpretation: it diffuses $u$ is in the direction orthogonal to its gradient $\nabla u$ and does not diffuse at all in the direction of the gradient $\nabla u$.

Weickert $[10,11,9]$ proposed a fully anisotropic diffusion filter. It is obtained by integrating a $1 \mathrm{D}$ diffusion equation in the direction of $e_{\phi}=(\cos \phi, \sin \phi)^{T}$ over $\left[0,180^{\circ}\right]$ :

$$
\begin{array}{r}
\frac{\partial u}{\partial t}=\lambda \int_{0}^{\pi} \frac{\partial}{\partial e_{\phi}}\left(g\left(\left|\frac{\partial G_{\sigma} * u}{\partial e_{\phi}}\right|\right) \frac{\partial u}{\partial e_{\phi}}\right) d \phi \\
u(x, y, 0)=u_{0}(x, y),
\end{array}
$$

where the boundary condition is given by

$$
\frac{\partial}{\partial e_{n}}\left(g\left(\left|\frac{\partial G_{\sigma} * u}{\partial e_{n}}\right|\right) \frac{\partial u}{\partial e_{n}}\right)=0 .
$$

This is a true anisotropic diffusion process with a diffusion tensor that prefers diffusion in the direction parallel to the edge to diffusion in the direction perpendicular to the edge.

As can be seen from the above, there have been several important conceptual advances in scale-space, from the linear scale-space proposed by Witkin and Koenderink [6, 12], first to nonlinear diffusion initiated by Perona and Malik [7], then to the anisotropic diffusion models by Lions and Morel's group [1] and by Weickert [9]. In this paper, we propose another conceptual advance motivated by difficulties with the current state-of-the-art of geometry-driven diffusion. All difficulties are associated with the diffusion coefficient/tensor that continuously depends on the gradient, and the resulting nonlinear diffusion equation.

1. The first difficulty is with the need to smooth the image before estimating the gradient. First, what should be the size of the Gaussian kernel $\sigma$

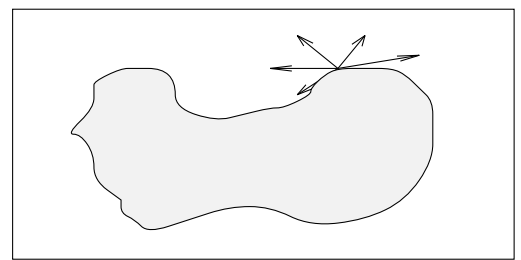

Figure 1: Desired anisotropy and asymmetry near edges. Diffusion should be stopped at edges, but allowed in all directions inside each region.

used to smooth the image? Details smaller than $\sigma$ will be eliminated by the smoothing Gaussian kernel. Since Gaussian smoothing is the same as nonselective linear diffusion, this process is equivalent to a nonselective linear diffusion before a selective nonlinear diffusion. The question is how long the first diffusion should go on before the second diffusion can start. This nonselective presmoothing becomes more damaging considering that the noise may not be uniformly distributed over the image and may be correlated with the signal. It smoothes every part of the image by the same amount, regardless of the amount of noise in that part.

2. Two forms of $g(s)$ often used are:

$$
g(s)=\exp \left(-s / K^{2}\right) \text { or } g(s)=\frac{1}{1+(s / K)^{2}}
$$

where the parameter $K$ must be chosen appropriately. A large $K$ will cause low contrast edges to be smoothed out, while a small $K$ leads to slow diffusion within regions with no edges. Slowly varying edges spanning a large area will be smoothed out if the gradient is lower than the threshold determined by $K$ in the beginning since the gradient will become even lower as the diffusion goes on.

3 . In the anisotropic diffusion processes in $[1,10$, 11, 9] (Eqs. (3) and (4)), the diffusion operators are symmetric, i.e., the diffusion is the same in directions $e_{\phi}$ and $-e_{\phi}$. In both cases, diffusion is not allowed in the direction orthogonal to the edge. However, what is really desired is to make the diffusion asymmetric at the edge such that diffusion in all directions within an region except across the edge are allowed, as shown in Fig. 1.

In this paper, we propose a diffusion process in Section 2 which solves the above difficulties and is simple to implement. Section 3 presents experimental results. Conclusion and discussion are given in Section 4 . 


\section{Local Scale Controlled Anisotropic Diffusion and Local Noise Estimate}

The new diffusion process is based on the following simple observation: Anisotropic, nonlinear diffusion equation using diffusion coefficients/tensors that continuously depend on the gradient is not necessary to achieve sharp, undistorted, stable edge detection across many scales. All is needed is to know when and where to stop the linear diffusion, i.e, a piecewise linear diffusion process rather than a nonlinear diffusion process. Diffusion should be full speed in all directions inside each region. It should stop at the edges, in different times at different parts of the image. This corresponds to an extension of the important idea of local scale control proposed by Elder and Zucker $[4,5]$.

An image may have a broad range of local blur and sizes of local structures. As a result, edges and structures at different part of an image may be best viewed at different scales. Local scale control $[4,5]$ determines a unique scale for local estimation at each point in an image. Like nonlinear diffusion, local scale control solves the problem of tracking features across multiscales.

\subsection{Local Scale Control with Local Noise Estimate}

The key to local scale control is the prior computation of a significance function $s(\sigma)$ which determines the lower bound on the smoothing kernel needed to reliably assert a non-zero gradient or to reliably determine the sign of the second order derivative. Reliability here means that the likelihood of making at least one decision error over an entire image is less than a given tolerance, say $5 \%$. The significance function depends on the $L_{2}$ norm of the filter, the statistics of the sensor noise, and the quantity to be estimated. For Gaussian i.i.d. sensor noise and an image of standard size $(\approx 256 \times 384)$, Elder and Zucker [4, 5] found the significance functions for asserting a non-zero gradient or for determining the sign of the second order derivative to be

$$
s_{1}\left(\sigma_{1}\right)=3 \sigma_{n} / \sqrt{\pi} \sigma_{1}^{2}, \quad s_{2}\left(\sigma_{2}\right)=5 \sigma_{n} / 2 \sqrt{2 \pi} \sigma_{2}^{3},
$$

where $\sigma_{n}$ is the standard deviation of the noise, $\sigma_{1}$ and $\sigma_{2}$ are the scales of the Gaussian first and second derivative filters respectively.

At the beginning, the gradient (second order derivative) response is normally smaller than the significance function. With increasing scale, both the gradient (second order derivative) response and the significance function decrease. However, the significance function decreases much faster than the gradient (second order derivative) response. The scale at which the two

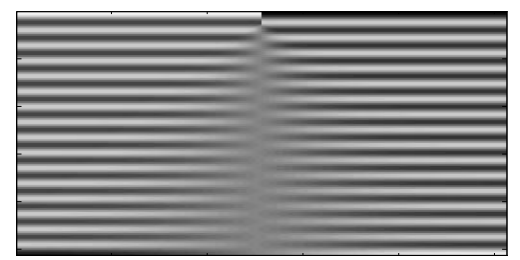

Figure 2: The blur of the vertical edge at the center increases gradually from top to bottom. The scale of the blur of the horizontal edges is much smaller and fixed. This shows that local scale control should be anisotropic.

curves intersect is the minimum reliable scale for asserting a nonzero gradient (zero-crossing of the second order derivative). Elder and Zucker estimated $\sigma_{n}$ from the image by highpass filtering a slow varying region of the image. They obtained the minimum reliable scale at each point by using the smallest scale at which a nonzero gradient or a zero-crossing of the second order derivative can be reliably detected, chosen from gradients or second order derivatives computed at a certain interval of scales, say $\sigma_{1}\left(\right.$ or $\left.\sigma_{2}\right)=0.5,1,2,4,8$. As demonstrated in $[4,5]$, local scale control enables the scale to be chosen according to the local contents of the image, and yields edge detection results that remove noises, show both the details and clear edges from blurred contours.

The local scale control as presented by Elder and Zucker [4, 5] needs further improvements.

1. The local scale as presented by Elder and Zucker is isotropic. The example in Fig. 2 shows the necessity of anisotropic local scale control. The blur scale of the horizontal edges is small and constant while the blur scale for the vertical edge varies over a wide range.

2. A more important problem with the local scale as presented by Elder and Zucker is that the same noise variance $\sigma_{n}^{2}$ is used for the entire image. This assumes that the noise is uniformly distributed over the image and uncorrelated with the image signal. This assumption is not true in general. The example shown in Fig. 3 is a 1D signal obtained from a single row of the clown image (60th row in Fig. 4(a)). It is obvious that the noise is not uniformly distributed. If a single noise variance estimate is used in the local scale, places with less noise will be oversmoothed, while places with more noise will be undersmoothed. In $2 \mathrm{D}$ images, the noise variance at a point may be different in each direction. 
To overcome these problems, it is necessary to estimate the noise at each point in each direction. There may be several feasible noise estimates. We propose a noise estimate based on the number of zero-crossings of the second order derivatives in a direction and the amount of unipolar energy in the directional derivative, i.e.,

$$
\sigma_{n}\left(i, j, e_{\phi}, \beta, \gamma\right)=\beta \frac{Z\left(i, j, e_{\phi}, \beta, \gamma\right)}{1+\left|\sum_{(i, j)}^{(i, j)+\gamma e_{\phi}} \frac{\partial u}{\partial e_{\phi}}\right|}
$$

where $Z\left(i, j, e_{\phi}, \beta, \gamma\right)$ is the number of zero-crossings of the second order derivative at $(i, j)$ in the direction $e_{\phi}$ over the distance of $\gamma$, i.e., from $(i, j)$ to $(i, j)+\gamma e_{\phi}$. $\beta$ and $\gamma$ can also be estimated from the image. This estimate is motivated by the following two facts:

1. In a place with lots of noise, there is always lots of zero-crossings of the second order derivative. Therefore, the noise estimate should increase with $Z\left(i, j, e_{\phi}, \beta, \gamma\right)$.

2. A significant edge is associated with a large, mostly unipolar, i.e, either mostly positive or mostly negative, derivative over a certain range. In this case, the sum $\sum_{(i, j)}^{(i, j)+\gamma e_{\phi}} \frac{\partial u}{\partial e_{\phi}}$ in the denominator will be large. On the other hand, in a region with high frequency noise over a slowly varying signal, the derivative will oscillate between positive and negative. Thus, the sum $\sum_{(i, j)}^{(i, j)+\gamma e_{\phi}} \frac{\partial u}{\partial e_{\phi}}$ will be small. As a result the denominator reduces the noise estimate at high contrast edges to avoid smoothing out the edge, and at the same time, maintains the high noise estimate level at regions with heavy noise over a slowly varying signal.

In our implementation, four directional noise estimates is computed at each point:

$$
\begin{gathered}
s_{1}\left(\sigma_{1}, i, j, e_{\phi}, \beta, \gamma\right)=\sigma_{n}\left(i, j, e_{\phi}, \beta, \gamma\right) / \sigma_{1}^{2}, \\
s_{2}\left(\sigma_{2}, i, j, e_{\phi}, \beta, \gamma\right)=\sigma_{n}\left(i, j, e_{\phi}, \beta, \gamma\right) / \sigma_{2}^{3}, \\
e_{\phi}=0, \pi / 2, \pi, 3 \pi / 4
\end{gathered}
$$

The constants in Eq. (7) are incorporated in the parameter $\beta$. Note that this estimation needs to be done only once before the diffusion starts. Figs. $3(\mathrm{~b})$ and (c) show the two directional noise estimates of the signal in Fig. 3(a) with $\beta=1$ and $\gamma=5$ points in both directions. Observe that both noise estimates are very small around the significant edges and large at places with lots of oscillations.

\subsection{Local Stopping of Linear Diffusion with Anisotropic and Asymmetric Diffusion at Edges}

In the local scale control proposed by Elder and Zucker $[4,5]$, the image is smoothed with a set of linear scale filters. The results from multiple scales are combined into a single local scale image. Based on the equivalence of scale space and diffusion, we propose a new type of local scale control process: local stopping of a linear diffusion process, i.e., a linear diffusion process that stops at different times at different places. The result is a single image. We will show that the new local scale controlled diffusion will lead to a much simpler algorithm but with the desired properties of geometry-driven anisotropic, asymmetric nonlinear diffusion. The new diffusion process does not need a continuous gradient dependent diffusion rate/tensor. It is a linear diffusion process with local stopping criteria. Diffusion is full speed in all directions inside a region while stops at the edges, in different times at different parts of the image.

This is achieved with a simple modification of Eq. (4) by replacing the gradient dependent function $g\left(\left|\frac{\partial G_{\sigma} * u}{\partial e_{\phi}}\right|\right)$ with a piecewise linear diffusion rate:

$$
\begin{array}{r}
\frac{\partial u}{\partial t}=\lambda \int_{0}^{2 \pi} \frac{\partial}{\partial e_{\phi}} \\
\left(g\left(\frac{\partial^{n} u_{\sqrt{t}}}{\partial e_{\phi}^{n}}, s_{n}\left(\sqrt{t}, i, j, e_{\phi}, \beta, \gamma\right)\right) \frac{\partial u}{\partial e_{\phi}}\right) d \phi
\end{array}
$$

where $g\left(\frac{\partial^{n} u_{\sqrt{t}}}{\partial e_{\phi}^{n}}, s_{n}\left(\sqrt{t}, i, j, e_{\phi}, \beta, \gamma\right)\right)$ is a binary function of the derivative $\frac{\partial^{n} u}{\partial e_{\phi}^{n}}$, the significance function $s_{n}\left(\sqrt{t},, i, j, e_{\phi}, \beta, \gamma\right), \mathrm{n}=1$ or 2 , and the scale $\sqrt{t}$ :

$$
\begin{array}{r}
g\left(\frac{\partial u_{\sqrt{t}}^{n}}{\partial e_{\phi}^{n}}, s_{n}\left(\sqrt{t}, i, j, e_{\phi}, \beta, \gamma\right)\right)= \\
\begin{cases}1 & \text { if }\left|\frac{\partial u^{n} \bar{t}}{\partial e_{\phi}^{n}}\right|<s_{n}\left(\sqrt{t}, i, j, e_{\phi}, \beta, \gamma\right) \quad n=1,2 . \\
0 & \text { otherwise }\end{cases}
\end{array}
$$

The term $u_{\sqrt{t}}$ means that the image $u$ has diffused for time $t$, or equivalently been smoothed by Gaussian kernel $\sqrt{t}$, modulated by the binary function $g(\bullet, \bullet)$. The initial and boundary conditions are the same as in Eqs. (4) and (5), i.e., diffusion along the normal of the image boundary is zero. Note that the integral in Eq. (10) is over the interval $(0,2 \pi)$ so that diffusion can be asymmetric on two sides of the edges.

The new local scale controlled diffusion can be based ei- 
ther on the magnitude of the gradient using the diffusion function $g\left(\frac{\partial u}{\partial e_{\phi}}, s_{1}\left(\sqrt{t}, i, j, e_{\phi}, \beta, \gamma\right)\right)$, or on the zero-crossing of the second order derivative using the diffusion function $g\left(\frac{\partial^{2} u}{\partial e_{\phi}^{2}}, s_{2}\left(\sqrt{t}, i, j, e_{\phi}, \beta, \gamma\right)\right)$. The diffusion starts with $g\left(\frac{\partial^{n} u_{0}}{\partial e_{\phi}^{n}}, s_{n}(0)\right)=1$ for all pixels, where either $n=1$ or $n=2$. This is linear diffusion for the entire image. As time goes by, the significance function $s_{1}\left(\sqrt{t}, i, j, e_{\phi}, \beta, \gamma\right)=\sigma_{n}\left(i, j, e_{\phi}, \beta, \gamma\right) / t$, or $s_{2}\left(\sqrt{t}, i, j, e_{\phi}, \beta, \gamma\right)=\sigma_{n}\left(i, j, e_{\phi}, \beta, \gamma\right) / \sqrt{t^{3}}$ declines much faster than the response of the gradient filter or the second order derivative filter. For local scale controlled diffusion based on the magnitude of the gradient, set the function $g\left(\frac{\partial u_{\sqrt{t}}}{\partial e_{\phi}}, s_{1}\left(\sqrt{t}, i, j, e_{\phi}, \beta, \gamma\right)\right)=0$ at the next pixel in the $e_{\phi}$ direction as soon as the magnitude of gradient at that pixel exceeds the significance function $s_{1}\left(\sqrt{t}, i, j, e_{\phi}, \beta, \gamma\right)=\sigma_{n}\left(i, j, e_{\phi}, \beta, \gamma\right) / t$. For local scale controlled diffusion based on zero-crossing of the second order derivative, set the function $g\left(\frac{\partial^{2} u}{\partial e_{\phi}^{\sqrt{t}}}, s_{2}\left(\sqrt{t}, i, j, e_{\phi}, \beta, \gamma\right)\right)=0$ at the next pixel in the $e_{\phi}$ direction as soon as a zero-crossing of the second order derivative at that pixel can be reliably detected, i.e., as soon as the magnitudes of the second order derivative at neighboring pixels exceed the significance function $s_{2}\left(\sqrt{t}, i, j, e_{\phi}, \beta, \gamma\right)=\sigma_{n}\left(i, j, e_{\phi}, \beta, \gamma\right) / \sqrt{t^{3}}$, and there is a sign change of the second order derivative. This stops the diffusion either at pixels with significant magnitude of gradient or at a zero-crossing in a direction as soon as the gradient or zero-crossing can be reliably detected in that direction. After a sufficient amount of time, diffusion is stopped along all boundaries with significant gradient or second order derivative zero-crossings exceeding the significance function. In regions inside boundaries with significant gradient or bounded by zero-crossings of the second order derivative, diffusion is still linear except at the boundary. On the boundary, the diffusion is zero. Next to the boundary, the diffusion is anisotropic, asymmetric, and does not cross the boundary as illustrated in Fig. 1. The anisotropy and asymmetry is only with respect to the boundary in the sense that diffusions on two sides of the boundary are totally independent. This is a much more efficient scheme for smoothing inside a region than the methods by Lions and Morel's group [1], and by Weickert [10, 11, 9] where diffusion near the boundary is only concentrated along the direction of the boundary.

Advantages of the new local scale controlled diffusion algorithm is summarized below:

1. It stops the diffusion at the place and time de- termined by the significance function using a spatial variant, anisotropic local noise estimate. The use of local noise estimate avoids nonselective smoothing, and further enhances selective smoothing. Together with local stopping, it solves the difficulty of determining the size of Gaussian kernel $\sigma$ for smoothing the image in order to reliabley estimate the derivatives, existing in previous geometry-driven diffusion algorithms.

2. It advances the local scale concept by introducing spatial variant, anisotropic local noise estimation and local stopping of diffusion. The local noise estimate is more realistic than the previous assumption of uniformly distributed noise uncorrelated with the image signal. The local noise estimate significantly improves local scale control. With the local noise estimate, heavy noise areas are smoothed substantially more, and at the same time, enables preservation of sharper significant edges, than a constant noise estimate for the entire image. Local stopping of diffusion allows an efficient implementation of local scale control.

3. It not only does not diffuse across boundaries of significant gradient or zero-crossings of the second order derivative, but also enhances edges. Unlike in $[7,8]$ where the edges are enhanced right from the beginning, which could amplify noises, our algorithm starts to enhance edges only after just the right amount of smoothing specified by the significance function and the local noise estimate. This is illustrated in Fig. 3 using a 1D example. Notice that the edges in Fig. 3 become shaper after the diffusion. As soon as a zero-crossing of the second order derivative can be reliably detected according to the significance function, the diffusion is stopped at the zero-crossing. Since the diffusion form the two sides keep on diffusing towards the zero-crossing point where the diffusion is stopped, it brings one side higher, and the other side lower, thus, enhances the edge. Because of the fixed boundary condition used, there will be no bunching up at the place where diffusion is stopped. The points where diffusion is stopped will eventually reach steady state, i.e., be brought up or down to the same level of immediate edges, if the diffusion keeps on going. Afterwards, there will be no more changes because gradients will be zero everywhere except at the points where diffusion is stopped. In experiments, it is found that for most images, after about 20 to 50 iterations, it will be very close to the steady state and changes 


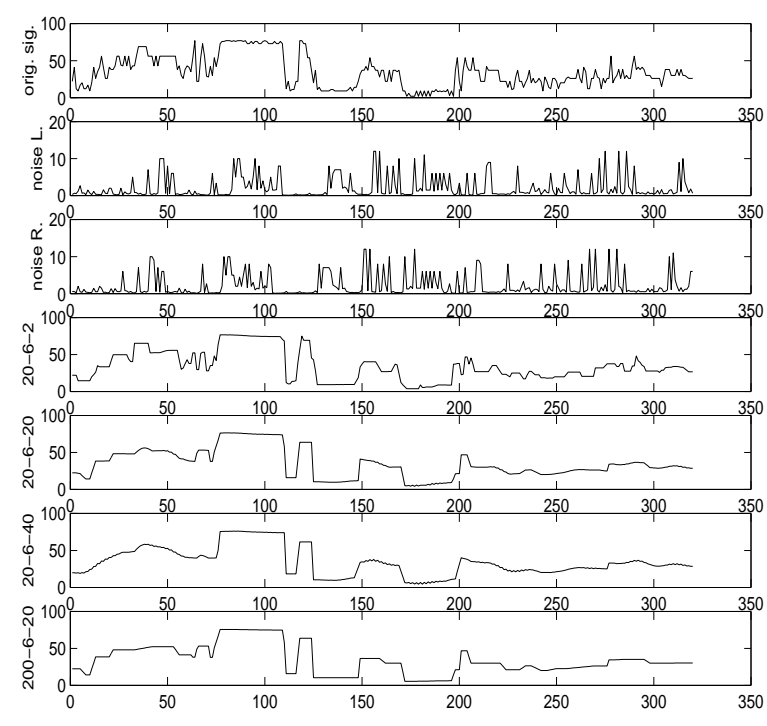

Figure 3: The first plot is a noisy and blurred edge. It is the 60th row of the original clown image shown in Fig. 4(a). The second and third plots show the local noise estimate as defined in Eq. (8) with $\beta=1$ for in the left and right directions respectively. The remaining plots show the diffusion results. The three numbers in the format of a-b-c next to the vertical axes indicate: $\mathrm{a}$ - the number of iterations, $\mathrm{b}$ - the range $\gamma$ in which the local noise is estimated, and c - the value of $\beta$ used. The result in the last plot is obtained with the same $\beta$ and $\gamma$ as the fifth plot. The only difference is the fifth plot is obtained with 20 iterations, while the last one is obtained with 200 iterations. As can be seen, the steady state is approximately reached after a small number of iterations.

become very small for further diffusion.

In previous geometry-driven nonlinear diffusion, although diffusion across edges is small, it is not zero. If the diffusion is allowed a long period of time, some edges will be smoothed out. On the other hand, the local stopping in our approach, the steady state is reached approximately in a small number of iterations. Afterwards, even if the diffusion continues to run for a long period of time, it will not smear edges, but simply make the edges sharper.

4. The algorithm is very simple to implement, and only two parameters are required: the scale factor $\beta$ of the noise estimate $\sigma_{n}$ and the $\gamma$ used for counting the number of zero-crossings. In addition, there is no need to first smooth the im- age nonselectively before the selective geometrydriven diffusion as in previous algorithms. There is only one diffusion process, thus only one copy of the image is needed in our algorithm.

5. It will enhance a slowly varying edge that persists long enough in the diffusion process even if the estimate of $\sigma_{n}$ is too large, because the significance function declines much faster than the gradient and second order derivative filters. A slowly varying edge will remain to exist for a long time in the diffusion process if it spans a long range. The ability to enhance slowly varying edges is in contrast to previous geometry-driven algorithms. In these algorithms, if a large $K$ (Eq. (6)) is chosen and if in the beginning the gradient of a slowly varying edge spanning a long range is smaller than the threshold determined by $K$, it will become even lower as the diffusion goes on. As a result, these slowly varying edges tend to be smoothed out .

6. It is anisotropic and asymmetric only with respect to boundaries with significant gradient or reliably detected zero-crossings of the second order derivative, thus allowing diffusion in all directions on each side of the edge, and leading to simple computation and fast smoothing within a region. In previous geometry-driven diffusion algorithms, diffusion is the same in direction $e_{\phi}$ and $-e_{\phi}$, and diffusion near the boundary is concentrated to be parallel to the edge.

What edges get enhanced at what time is determined by the the local estimate of the amount of noise in the image, i.e., $\sigma_{n}$ in the significance function. If $\beta$ in $\sigma_{n}$ is chosen too small, some small variations will be enhanced as well, leading to the staircase phenomenon similar to those in other geometry-driven methods as pointed out in [8]. These staircases may be eliminated by increasing $\beta$. If $\beta$ is chosen too large, small structures or low contrast edges may be smoothed out. However, if the low contrast edge extends a longer range than the smaller variations, and can survive a longer diffusion time, it will eventually be enhanced.

\section{Experimental Results}

We implemented two versions of the new local scale controlled diffusion. One based on the magnitude of the gradient and the other based on the zero-crossing of the second order derivative. Some results are presented in this section. For comparison, results using the improved Perona and Malik diffusion using $g\left(\left|\nabla G_{\sigma} * u\right|\right)$ in Eq. (2), as proposed in [3], are also given. In the improved Perona and Malik diffusion, 


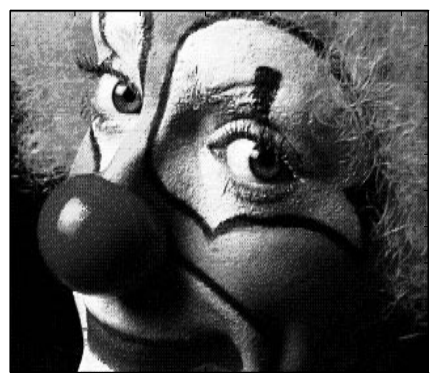

(a)

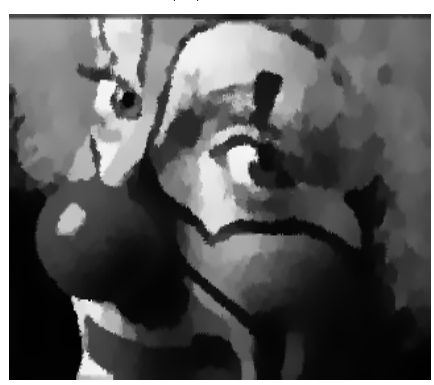

(c)

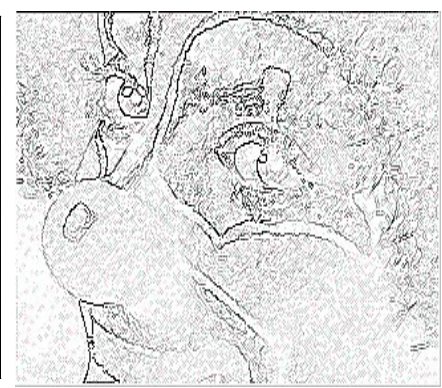

(b)

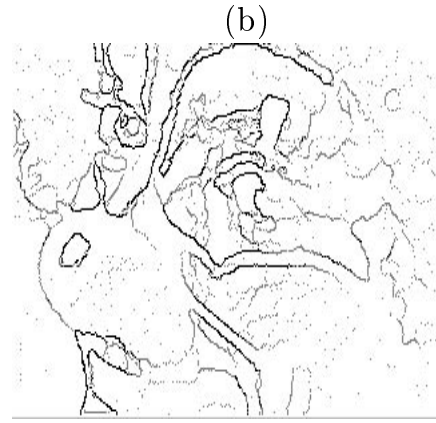

(d)
Figure 4: (a) Original clown image. (b) Edge detection result of the original clown image. (c) Local scale controlled diffusion based on zero-crossing of the second order derivative, $\beta=100, \gamma=6,60$ iterations. (d) Edge detection result of the diffused image in (c).

there are three parameters: $\sigma$ in $g\left(\left|\nabla G_{\sigma} * u\right|\right), K$ in Eq. (6), and the number of iterations. $G_{\sigma}=G_{1}$ in all examples using the improved Perona and Malik diffusion. Smaller $\sigma$ leads to noisy edge detection results, requiring more iterations to remove noises. Bigger $\sigma$ means more nonselective smoothing, leading to loss of more details. In our algorithm, there are also three parameters: $\beta, \gamma$, and the number of iterations. However, the number of iterations is unimportant in our algorithms as discussed previously.

In all cases, the edge images are obtained using the Canny edge operator [2] on the Web at http://www.ius.cmu.edu/demos/edge.html provided by Henry Rowley of CMU. In this implementation, the Gaussian smoothing kernel is a $3 \times 3$ window with $\sigma \approx 0.6$. Therefore, smoothing is minimal and the algorithm basically detects maximums of the gradients.

Fig. 4 shows the $200 \times 320$ clown image in the Matlab, examples of the diffused clown images using the new local scale controlled diffusion and the improved Perona and Malik diffusion with similar amount of diffusion, and the edge detection results using the diffused images. Different parameter settings were used in each case.
Figs. 5 shows the Canaletto image used in $[7,8]$, examples of diffusion of the image using the new local scale controlled diffusion and the improved Perona and Malik diffusion with similar amount of diffusion, and the edge detection results using the diffused images. The size of the Canaletto image is $479 \times 512$. The image has a broad range details, blurs and contrasts.

In the experiments it is observed that the new local scale control diffusion yields comparable or better results compared to the improved Perona and Malik diffusion, but with an algorithm that is simpler and easier to analyze. Quantitative study with specified applications is required for a more accurate assessment.

Note that in both Figs. 4 and 5 , the $\beta$ used in the zero-crossing stopping criterion is larger that required in the magnitude of gradient based criterion to obtain similar results. This is because the zero-crossing criterion is more strict than the magnitide of gradient criterion, and is able to eliminate more unwanted noises. The advantage of the magnitude of gradient based criterion is that it is simpler to compute and faster to run.

\section{Conclusion and Discussion}

We showed that piecewise linear diffusion may be a better alternative than nonlinear anisotropic diffusion for image smoothing and edge detection. The spatial variant, anisotropic local noise estimate we proposed advances the important idea of local scale control by Elder and Zucker $[4,5]$. Our approach of local stopping of diffusion offers an efficient implementation of local scale control. Our local scale controlled diffusion based on the local noise estimate determines a unique scale for local estimation at each point in an image by stopping at the right place at the right time. It produces a single image that is selectively smoothed with edges enhanced and accurately localized, making tracking features across multiscales unnecessary.

Local scale controlled diffusion does not diffuse across reliably detected significant gradients or reliably detected zero-crossings of the second order derivative. It is anisotropic and asymmetric only with respect to reliably detected edges. This allows diffusion in all directions on each side of the edge, leading to simple computation and fast smoothing within a region. It also enhances slowly varying edges that persist long enough in the diffusion process because of the declination of the significance function. These two points are difficult to achieve with previous geometrydriven diffusion algorithms.

Future work includes investigation of other local noise estimates, methods for estimating $\beta$ and $\gamma$ from 


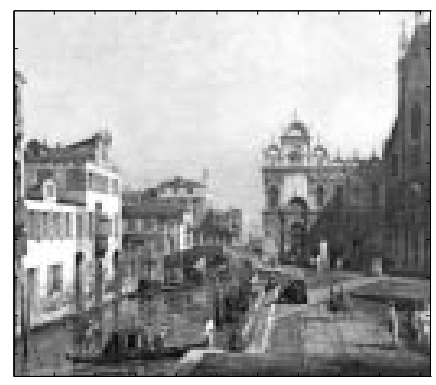

(a)

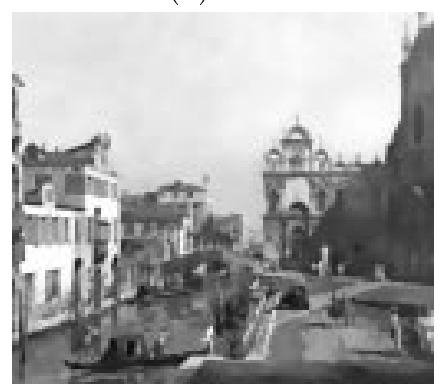

(c)

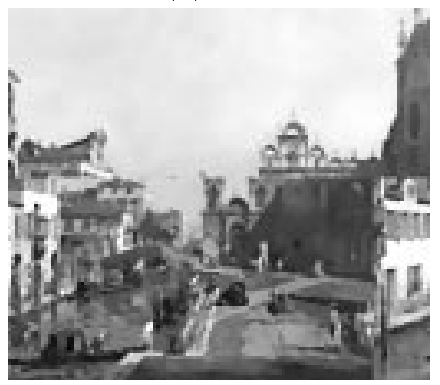

(e)

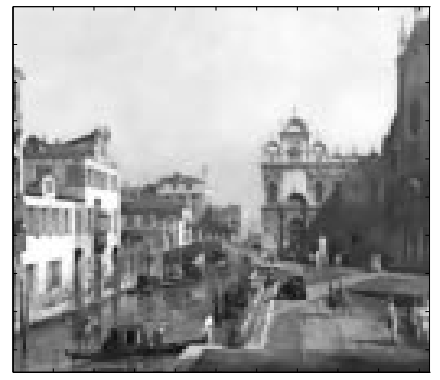

(g)

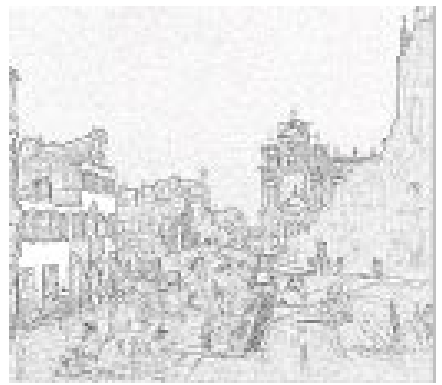

(b)

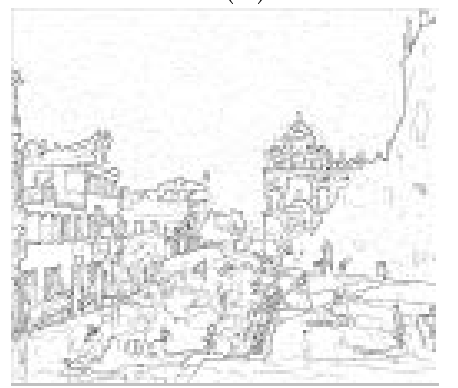

(d)

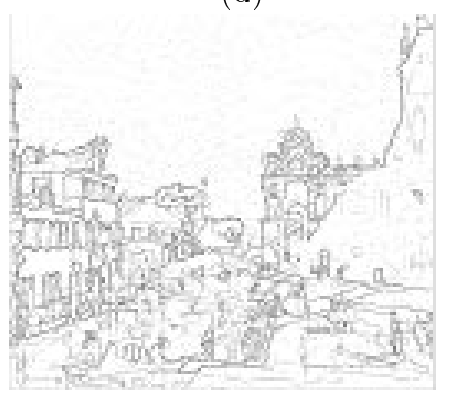

(f)

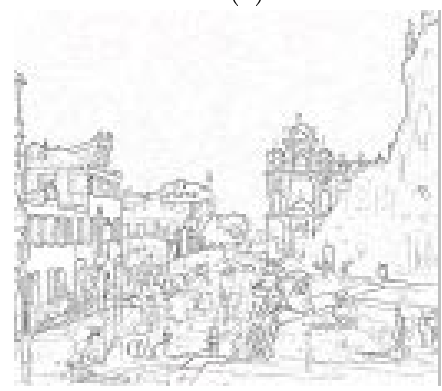

(h)
Figure 5: (a) Original Canaletto image. (b) Edge detection result of the original Canaletto image. (c) Local scale controlled diffusion based on magnitude of gradient, $\beta=200, \gamma=6,50$ iterations. (d) Edge detection result of the diffused image in (c). (e) Local scale controlled diffusion based on zero-crossing of the second order derivative, $\beta=80, \gamma=6,50$ iterations. (f) Edge detection result of the diffused image in (e). (g) Improved Perona and Malik diffusion with $G_{\sigma}=G_{1}, K=4,60$ iterations. (h) Edge detection result of the diffused image in $(\mathrm{g})$. images, and quantitative evaluation of the new local scale controlled diffusion, in comparison with other geometry-driven diffusion and edge detection methods.

\section{References}

[1] L. Alvarez, P.L. Lions, and J.M. Morel. Image selective smoothing and edge detection by nonlinear diffusion. ii. SIAM J. Numer. Anal., 29, 1992.

[2] J. Canny. A computational approach to edge detection. IEEE Trans. on Pattern Analysis and machine Intelligence, 8, 1986.

[3] F. Catte, T. Coll, P.L. Lions, and J.M. Morel. Image selective smoothing and edge detection by nonlinear diffusion. SIAM J. Numer. Anal., 29, 1992.

[4] J.H. Elder and S.W. Zucker. Local scale control for edge detection and blur estimation. In Proc. 4th Euro. Conf. on Comp. Vis., 1996.

[5] J.H. Elder and S.W. Zucker. Scale space localization, blur, and contour-based image coding. In Proc. IEEE CVPR, 1996.

[6] J. Koenderink. The structure of images. Biol. Cybern., 50, 1984.

[7] P. Perona and J. Malik. Scale-space and edge detection using anisotropic diffusion. IEEE Trans. on Patt. Anal. and Mach. Intell., 12, 1990.

[8] P. Perona, T. Shiota, and J. Malik. Anisotropic diffusion. In B.M. ter Harr Romeny, editor, Geometry-Diffusion in Computer Vision. Kluwer Academic Publishers, 1994.

[9] J. Weickert. Anisotropic diffusion filters for image processing based quality control. In Proc. 7th Euro. Conf. on Math. in Industry, 1994.

[10] J. Weickert. Nonlinear diffusion scale-space: From the continuous to the discrete setting. In M.O. Berger et. al., editor, ICAOS '96: Images, Wavelets and PDEs., volume 219, 1996.

[11] J. Weickert. Theoretical foundations of anisotropic diffusion in image processing. In W. Kropatsch et al., editor, Theoretical Foundations of Computer Vision. Computing Suppl. 11, 1996.

[12] A. Witkin. Scale-space filtering. In Proc. Intl. Joint Conf. on Artificial Intelligence, Karlsruhe, West Germany, 1983. 